\title{
Diagnóstico do linfedema: análise dos métodos empregados na avaliação do membro superior após linfadenectomia axilar para tratamento do câncer de mama
}

\author{
Diagnosis of Iymphedema: analysis of the methods used in the evaluation of \\ the upper limb in women undergoing axillary linfadenectomy for breast can- \\ cer treatment
}

Anke Bergmann'1 Inês Echenique Mattos², Rosalina Jorge Koifman³

\begin{abstract}
Resumo
Introdução: 0 câncer de mama é a principal neoplasia que acomete as mulheres e sua principal complicação é o linfedema, que causa importantes alterações físicas, emocionais, sociais e psicológicas. 0 seu diagnóstico precoce deve ser preconizado, sendo necessário estabelecimento de critérios adequados e factíveis para sua mensuração. O bjetivos: Avaliar a concordância e a validade dos métodos subjetivos e objetivos empregados no diagnóstico do linfedema. M etodologia: Foram estudadas 394 mulheres submetidas a tratamento cirúrgico para câncer de mama, entre abril e agosto de 2000. O s dados foram obtidos por entrevista, exame físico e revisão de prontuários. Foram utilizados métodos objetivos (perimetria, volume estimado do membro) e subjetivos (relato de inchaço e de sintomas de edema, anotações em prontuário). D eterminou-se a concordância simples, sensibilidade, especificidade e os valores preditivos positivo e negativo entre os métodos. Resultados: A prevalência de linfedema variou entre $11,5 \%$ e $30,7 \%$. 0 volume estimado $>300 \mathrm{ml}$ e a perimetria $>3,00 \mathrm{~cm}$ apresentaram melhor concordância entre os critérios objetivos ( $96 \%$ ). Considerando como padrão-ouro o volume estimado do membro $=200 \mathrm{ml}$, a perimetria com ponto de corte de $2,5 \mathrm{~cm}$ foi o método com melhor relação sensibilidade/especificidade $(S=0,74 ; E=0,98)$. Conclusão: $0 \mathrm{~s}$ métodos com melhor concordância foram o volume estimado do membro $=300 \mathrm{ml}$ e a perimetria $=3,00 \mathrm{~cm}$. Considerando como padrão-ouro o volume estimado do membro $=200 \mathrm{ml}$, a perimetria com ponto de corte de $2,5 \mathrm{~cm}$ foi o método com melhor relação sensibilidade/especificidade $(S=0,74 ; E=0,98)$.
\end{abstract}

Palavras-chave: Neoplasias mamárias; Linfedema; Diagnóstico; Fisioterapia; Linfadenectomia axilar.

\footnotetext{
${ }^{1}$ Fisioterapeuta, M estre em Ciências da Saúde, Serviço de Fisioterapia, H ospital do Câncer III / Instituto N acional de Câncer / M S; D epartamento de Epidemiologia e M étodos Q uantitativos em Saúde, Escola N acional de Saúde Pública / FIO CRUZ / M S

2 M édica, D outor em M edicina

${ }^{3}$ M édica, D outor em Saúde Pública

Endereço para correspondência: Anke Bergmann - Rua Visconde de Santa Izabel, 274. Serviço de Fisioterapia / H CIII / IN CA. E.mail: abergmann@inca.gov.br, fisio.hc3@inca.gov.br.
} 


\begin{abstract}
Introduction: Breast cancer is the main neoplasia in women and its main complication is lymphedema, that causes important physical, emotional, social and psychological alterations. An important feature of the treatment of lymphedema is early diagnosis and to do so it is necessary the establishment of appropriate and feasible criteria for its mensuration. Purpose: To assess the agreement and the validity of subjective and objective methods used for lymphedema diagnosis. M ethods: A study was conducted with 394 women undergoing surgical treatment of breast cancer between April and August of 2000. The data were obtained by interview, physical examination and revision of medical records. O bjective methods (circumference, volume estimation) and subjective (report of swelling and edema symptoms, medical registries) were used. Simple agreement, sensibility, specificity and positive and negative predictive values were calculated in order to compare the different methods. Results: Lymphedema prevalence ranged from $11.5 \%$ to $30.7 \%$. The estimated volume $>300 \mathrm{ml}$ and the circumference $>3.00 \mathrm{~cm}$ presented better agreement among objective criteria (96\%). Considering the estimated volume of $200 \mathrm{ml}$ as the gold standard, circumference with a cut off point of $2.5 \mathrm{~cm}$ presented the best sensitivity/specificity ratio $(S=0.74 ; E=0.98)$. Conclusion: The methods presenting better agreement were $=300 \mathrm{ml}$ estimated volume of the limb and $=3.00 \mathrm{~cm}$ circumference. Considering the estimated volume of the limb $=200 \mathrm{ml}$ as the gold standard, perimetry with a cutoff point of $2,5 \mathrm{~cm}$ was the method presenting the best sensitivity/specificity ratio $(S=0.74 ; E=0.98)$.
\end{abstract}

Key words: Breast neoplasms; Lymphedema; Diagnosis; Physiotherapy; Axillary lymphadenectomy.

\section{INTRO DUÇÃO}

0 câncer de mama é um importante problema de saúde pública no Brasil, sendo a neoplasia mais freqüente entre as mulheres e correspondendo à principal causa de óbitos por câncer nesse grupo populacional. N as capitais brasileiras, as estimativas para 2003 das taxas brutas de incidência e mortalidade do câncer de mama por 100.000 mulheres são, respectivamente, 80,22 e 17,80 .

$\mathrm{N}$ a população brasileira, a maioria dos diagnósticos de câncer de mama é realizada em estádios tumorais mais avançados, onde se faz necessário instituir tratamentos mais radicais, com aumento significativo da morbidade e pior qualidade de vida. Em Belo H orizonte, no período de 1979 a 1996, 49\% dos diagnósticos de câncer de mama foram realizados no estádio II e 39\% no estádio III². Em estudo publicado por Palmeira et $\mathrm{a}^{3}$, entre os anos de 1996 e 1999, 95\% dos diagnósticos foram nos estádio II e III sendo, respectivamente, $48,5 \%$ e $46,5 \%$. No Rio de Janeiro, no ano de 1993, segundo dados do Registro $\mathrm{H}$ ospitalar de Câncer ${ }^{4}, 20,7 \%$ a 37,3\% dos casos de câncer de mama tratados nos hospitais do IN CA foram realizados no estádio II, e 32,3\% a 35,8\% no estádio III.

0 linfedema de membro superior é a principal complicação decorrente do tratamento do câncer de mama, sendo definido como um acúmulo de linfa nos espaços intersticiais, causado pela destruição dos canais de drenagem axilar, provocados pela cirurgia e/ou radioterapia $a^{5}$ ou ainda pela progressão locoregional da doença ${ }^{6}$. Segundo Stanton et $\mathrm{al}^{7}$, após a obstrução linfática, os coletores linfáticos do braço necessitam trabal har com uma resistência aumentada, e a instalação do edema dependerá da fadiga e do fracasso de bombeamento dos vasos linfáticos. Q uando instalado, causa importantes alterações físicas, psicológicas e sociais, que afetam a qualidade de vida das pacientes tratadas para câncer demama ${ }^{6,8,9} .0$ volume do membro, quando não tratado, aumenta progressivamente, assim como aumenta a freqüência das complicações a ele relacionado ${ }^{10,11}$, pois o acúmulo de linfa leva a estagnação de proteínas e conseqüente fibrose, tornando-se um meio de cultura propício para o desenvolvimento de linfangites eerisipelas, condições estas que agravam ainda mais o sistema linfático, previamente danificado ${ }^{12}$.

A prevalência do linfedema varia entre os estudos realizados, dependendo do tempo de seguimento, dos tratamentos realizados, da classificação e dos critérios utilizados para definição de linfedema. D e acordo com Petrek e $\mathrm{H}$ eelan ${ }^{13}$, de cerca de dois milhões de mulheres no mundo que sobreviveram ao câncer de mama, 15 a $20 \%$ estão vivendo atualmente com o linfedema decorrente do tratamento.

O linfedema é uma condição crônica que requer cuidado constante, podendo ocorrer de forma insidiosa em qualquer momento após o câncer de mama. 0 tratamento do linfedema visa a minimização e controle 
do volume do membro afetado, não sendo facilmente realizado e com resultado nem sempre satisfatório. Os tratamentos cirúrgicos e farmacológicos apresentam efeitos colaterais indesejáveis, e raramente são indicados ${ }^{14,15}$. Atualmente 0 tratamento preconizado é a terapia física complexa (Complex Physical Therapy) e suas variantes ${ }^{11,16-18}$.

Freqüentemente, o linfedema é diagnosticado através da anamnese, ou pelo exame físico ${ }^{19,20}$. D urante a anamnese, o diagnóstico subjetivo de linfedema pode ser obtido através do relato de sensação de braço "inchado" ou de sintomas sugestivos delinfedema, como a sensação que o braço está pesado, sensação de aperto em punho e dedos e a diminuição da flexibilidade da mão e cotovelo ${ }^{21}$. 0 exame físico compreende a inspeção, palpação e o emprego de técnicas objetivas para mensuração do linfedema. A inspeção deve ser realizada em todo o membro verificando-se: lesões cutâneas, fístulas e cistos linfáticos, micoses, amplitude de movimento, alterações ortopédicas associadas, entre outros. A palpação evidencia a consistência e a textura da pele, em busca de sinais de fibrose linfoestática, e ajuda na localização do edema. O s linfonodos regionais devem ser palpados para verificar a existência de metástases regionais $s^{11,18}$.

As principais técnicas objetivas utilizadas para 0 diagnóstico do linfedema, são a perimetria do membro em diferentes pontos, e as medidas volumétricas, obtidas ao submergir o membro em um cilindro com água ou pelo volume estimado através da circunferência do membro ${ }^{13,22,23}$.

As medidas de circunferência do membro (perimetria) são facilmente aplicadas na clínica, sendo realizadas em pontos fixos do membro. A medida volumétrica obtida pelo deslocamento de água ébaseada no princípio de Archimedes, onde o volume de água deslocado é igual ao volume do objeto imerso na água ${ }^{24}$. Segundo Boland e Adams $\mathrm{s}^{25}$, uma mudança de $10 \mathrm{ml}$ no volume do membro seria detectável ao usar esta técnica. Entretanto, na prática, este método apresenta várias desvantagens, pois o medidor é de difícil deslocamento entre os locais de exame, os recipientes são grandes e conseqüentemente demoram a encher e esvaziar, e não são indicados para pacientes com lesões de pele, e no período pós-operatório imediato.

Uma alternativa para se obter o volume do membro, mais adequado às condições existentes em nosso meio, consiste na obtenção de medidas de circunferência (perimetria). Algumas fórmulas geométricas têm sido empregadas para se obter o volume (cilindro, cone, retangular e trapezóide), sendo que a fórmula de cone apresentou melhores resultad $0 \mathrm{~s}^{26}$. Sander et $\mathrm{a}^{26}$ relataram boa confiabilidade intra e inter observador entre 0 volume de água e os volumes geométricos, em estudo realizado com 50 mulheres com linfedema de membro superior (ICC 0,91 e 0,99). Kaulesar et al ${ }^{27}$ encontraram coeficiente de correlação entreo volume de deslocamento de água e 0 volume calculado através da fórmula de cone maior que 0,90 .

A comparação entre a prevalência e/ou incidência de linfedema entre os diferentes estudos realizados é difícil, devido à utilização de diferentes critérios para diagnóstico, em populações distintas, submetidas a diferentes tratamentos, e com prognósticos variados.

Entre a população brasileira, espera-se uma freqüência elevada de linfedema, em conseqüência de tratamentos mais radicais do câncer devido ao diagnóstico tardio da doença. Sendo assim, os métodos demensuração do volume do membro para o diagnóstico do linfedema devem ser de fácil aplicação, de baixo custo, rápidos e seguros.

Este estudo tem como objetivo analisar a concordância e a validade de métodos utilizados para 0 diagnóstico de linfedema em mulheres submetidas a tratamento cirúrgico para câncer de mama, em um hospital público de referência.

\section{CASUÍSTICA E MÉTODOS}

Foram estudadas mulheres submetidas a tratamento cirúrgico para câncer de mama, em acompanhamento médico em um hospital público de referência, entre abril e agosto de 2000, que compareceram ao ambulatório de mastologia para consulta de seguimento. Foram excluídas as mulheres com câncer de mama bilateral; ausência de linfadenectomia axilar; doença ativa locorregional ou à distância; relato de al teração funcional em membros superiores anterior ao tratamento para câncer de mama; tratamento cirúrgico realizado há menos de 6 meses da data da entrevista; tratadas em outros hospitais; e aquelas que não apresentaram condições de responder às perguntas. As 394 mulheres que preencheram os critérios de inclusão foram informadas sobre os objetivos da pesquisa, e ao aceitar, assinaram o consentimento informado.

O s dados foram obtidos através de entrevista semiestruturada, exame físico e complementados através de revisão de prontuários. 0 exame físico foi realizado pela mesma profissional (A.B.) após padronização intraobservador de procedimentos, utilizando uma única fita métrica. A entrevista foi executada por outra pesquisadora, que desconhecia o status físico da paciente. A coleta das informações foi efetuada através 
de instrumentos padronizados e validados por meio da aplicação de um pré-teste em 20 mulheres em acompanhamento em outra instituição.

o linfedema foi diagnosticado utilizando-se os seguintes métodos:

\section{CRITÉRIOS OBjetIVOS}

- Circunferência: a medida da circunferência foi realizada em cinco pontos: a $21 \mathrm{~cm}$ e $11,5 \mathrm{~cm}$ acima do olecrano; e a 7,5 cm, $14 \mathrm{~cm}$ e $24 \mathrm{~cm}$ abaixo do olecrano ${ }^{28}$. A circunferência foi obtida, estando a paciente sentada, com o braço em abdução, antebraço fletido e a mão apoiada no tórax.

- Volume: 0 volume do membro foi estimado a partir das cinco medidas de circunferência, tratando cada segmento do membro como um par de circunferências (cone truncado). 0 volume do segmento foi dado por: $V=h .(C 2+C c+c 2) /(p, 12)$, onde $V$ é o volume do segmento do membro, $\mathrm{C}$ e $\mathrm{c}$ são as circunferências entre os pontos, e $h$ a distância entre as circunferências $(C, c) .0$ somatório da diferença entre cada ponto correspondeu ao volume final estimado ${ }^{29}$. 0 membro contralateral foi usado como controle.

\section{CRITÉRIOS SUBJETIVOS}

- Sintomatologia sugestiva: o linfedema foi considerado a partir de dois ou mais relatos da paciente de sintomas sugestivos de linfedema, sendo eles: sensação de peso no braço; sensação de pele esticada; diminuição da flexibilidade na mão, cotovelo ou ombro; e sensação de aperto no anel, relógio ou pulseira²1.

- Relato de edema: foi considerado linfedema o relato de inchaço no membro superior homolateral à mama operada após seis meses do tratamento cirúrgico.

- D iagnóstico em prontuário: o diagnóstico de linfedema em prontuário foi considerado a partir da existência de uma ou mais anotações de edema ou linfedema no membro superior homolateral, seis meses após o tratamento cirúrgico para câncer de mama.

A comparação dos métodos utilizados para diagnóstico de linfedema foi efetuada através da concordância simples. Para análise da validade de critério foi adotado como padrão-ouro para 0 diagnóstico de linfedema 0 volume indireto $=200 \mathrm{ml}$ do membro homolateral ao câncer de mama em comparação ao membro contralateral, estimado a partir da perimetria ${ }^{29}$. Avaliou-se a sensibilidade, visando testar a capacidade de diagnosticar corretamente as mulheres com linfedema; a especificidade, a fim de verificar a capacidade de determinar aqueles realmente sem linfedema; o valor preditivo positivo, para obter a proporção de linfedema entre as positivas ao exame diagnóstico; e o valor preditivo negativo, para verificar a proporção de sadios entre os negativos ao teste ${ }^{30}$.

C om a finalidade de demonstrar a inter-relação entre a sensibilidade e a especificidade e verificar qual 0 melhor ponto de corte a ser empregado na definição de linfedema, foi realizada a curva ROC (Receiver O perating $\mathrm{C}$ aracteristic). A área abaixo da curva RO C demonstra a acurácia do teste, podendo ser classificada como excelente $(0,90$ a 1,00$)$, boa $(0,80$ a 0,90$)$, satisfatória $(0,70$ a 0,80$)$, pobre $(0,60$ a 0,70$)$ ereprovada $(0,50 \text { a } 0,60)^{31}$.

Para análise dos dados foram utilizados os programas Epi-Info 6.04; SPSS 8.0, Stata 5.0 e PEPI 4.0.

Este estudo foi aprovado pela Comissão de Ética e Pesquisa do Instituto Nacional de Câncer e da Escola N acional de Saúde Pública da Fundação 0 swaldo Cruz. Todas as mulheres participantes do estudo assinaram 0 termo de consentimento informado.

\section{RESULTADO S}

A população estudada foi de 394 mulheres que apresentavam, na data da cirurgia, idade média de 55,2 anos, sendo o grupo etário de 50 a 59 anos o de maior freqüência (31\%). 0 tempo médio transcorrido da cirurgia até entrevista foi de 59 meses. Em média foram retirados 17 linfonodos, sendo classificados histologicamente como positivos $43 \%$. A média da diferença de volume estimado pela perimetria entre o membro afetado e o contralateral, foi $112,7 \mathrm{ml}$ (mediana $=68,28$; percentil $25=20,52$; percentil $75=172,46$ ) (tabela 1 ).

A casuística analisada era constituída por mulheres com câncer de mama com diagnóstico tardio, pois apenas 15\% foram diagnosticadas no estádio I. A maioria das mulheres foi submetida à mastectomia radical modificada (69\%). 0 registro no prontuário de linfadenectomia axilar completa (os três níveis axilares) foi o mais observado (60\%). Um número reduzido de mulheres da população em estudo foi submetida à reconstrução mamária imediata ou tardia (7\%). Q uanto ao tratamento radioterápico pós-cirúrgico, observamos que foi realizado em pouco mais da metade das mulheres ( $56 \%)$, sendo que destas, $17,6 \%$ também tiveram suas cadeias de drenagem linfática irradiadas. A quimioterapia foi realizada em $61,2 \%$ ea hormônioterapia com Tamoxifen em 44\% das mulheres (tabela 2).

A prevalência de linfedema na população do estudo variou entre $11,9 \%$ a $30,7 \%$, dependendo dos métodos utilizados no diagnóstico clínico e do ponto de corte empregado (tabela 3). 
Tabela 1 - Média, mediana e amplitude da variância de variáveis contínuas relacionadas ao linfedema.

\begin{tabular}{lccc}
\hline \multicolumn{1}{c}{ Variável } & Média & Mediana & $\begin{array}{c}\text { Amplitude de } \\
\text { variância }\end{array}$ \\
\hline Idade ao diagnóstico (anos) & 55,3 & 54 & $28-94$ \\
Seguimento (meses) & 59,5 & 46 & $7-287$ \\
Permanência com o dreno (dias) & 9,3 & 8 & $0-30$ \\
Número de linfonodos retirados & 17,2 & 16 & $2-48$ \\
Número de linfonodos comprometidos & 1,9 & 0 & $0-38$ \\
Índice massa corporal (IMC) & 27,4 & 27,3 & $16-44$ \\
Soma das diferenças do volume do membro $(\mathrm{ml})$ & 112,6 & 68,3 & $-362 / 1873$ \\
\hline
\end{tabular}

Tabela 2 - Características da população de estudo.

\begin{tabular}{|c|c|c|}
\hline Variásal & $N^{*}$ & $\%$ \\
\hline $\begin{array}{l}\text { Cirurgia realizada } \\
\text { Mastectomia a Halsted } \\
\text { Mastectomia radical modificada a Paley } \\
\text { Mastectomia radical modificada a } \\
\text { Madden } \\
\text { Canservodora }\end{array}$ & $\begin{array}{c}18 \\
203 \\
69 \\
101\end{array}$ & $\begin{array}{l}04,6 \\
51,5 \\
17,5 \\
26,6\end{array}$ \\
\hline $\begin{array}{l}\text { Linfodenectomia axila } \\
\text { Porcial (até nivel III } \\
\text { Total (oté nivel III) }\end{array}$ & $\begin{array}{c}237 \\
81\end{array}$ & $\begin{array}{l}60,2 \\
20,6\end{array}$ \\
\hline $\begin{array}{l}\text { Tratamento adjuvante } \\
\text { Quimioterapia } \\
\text { Radioterapia } \\
\text { Radioterapia (com cadeios de drenagem } \\
\text { linfótica) } \\
\text { Hormónioterapio }\end{array}$ & $\begin{array}{c}241 \\
221 \\
39 \\
172\end{array}$ & $\begin{array}{l}61,2 \\
56,1 \\
17,6 \\
43,7\end{array}$ \\
\hline $\begin{array}{l}\text { Estodiamento } \\
0 \\
1 \\
\text { II } \\
\text { III }\end{array}$ & $\begin{array}{c}5 \\
59 \\
209 \\
108\end{array}$ & $\begin{array}{l}01,3 \\
15,0 \\
53,1 \\
27,4\end{array}$ \\
\hline Reconstruçāo mamária & 28 & 07,1 \\
\hline
\end{tabular}

* A diferença nos percentuais refere-se a valores missing.

Tabela 3 - Prevalência de linfedema segundo diferentes métodos diagnósticos e pontos de corte empregados

\begin{tabular}{lcc}
\hline \multicolumn{1}{c}{ Critérios O bjetivos } & $\mathrm{N} *$ & $\%$ \\
\hline Perimetria $\geq 2,0 \mathrm{~cm}$ & 121 & 30,7 \\
Perimetria $\geq 2,5 \mathrm{~cm}$ & 70 & 17,8 \\
Perimetria $\geq 3,0 \mathrm{~cm}$ & 49 & 12,4 \\
& & \\
Volume $\geq 200 \mathrm{ml}$ & 82 & 20,8 \\
Volume $\geq 250 \mathrm{ml}$ & 62 & 15,7 \\
Volume $\geq 300 \mathrm{ml}$ & 47 & 11,9 \\
\hline \multicolumn{1}{c}{ Critérios subjetivos } & \\
\hline Sintomas sugestivos de linfedema & 91 & 23,1 \\
Relato de "inchaço" no braço & 120 & 30,5 \\
Prontuário médico & 64 & 16,2 \\
\hline
\end{tabular}

* A diferença nos percentuais refere-se a valores missing.

$\mathrm{Na}$ análise da concordância entre os métodos objetivos de diagnóstico de linfedema, observou-se concordância simples variando entre $74 \%$ e $96 \%$, segundo os diferentes pontos de corte analisados (tabela 4). 0 volume estimado $=300 \mathrm{ml}$ e a perimetria $=3,00$ apresentaram melhor concordância (96\%) e a menor concordância apresentada foi entre 0 volume estimado $=250 \mathrm{ml}$ e perimetria $=3,00 \mathrm{~cm}(74 \%)$.

Tabela 4 - Concordância dos métodos objetivos utilizados no diagnóstico de linfedema, segundo diferentes pontos de corte.

\begin{tabular}{|c|c|c|}
\hline $\begin{array}{l}\text { Volume estimado } \\
\text { prevoléncia } \mathrm{Py}\end{array}$ & Perimetria & $\begin{array}{l}\text { Concordancia } \\
\text { simples }\end{array}$ \\
\hline $\begin{array}{l}\geq 200 \mathrm{ml} \\
{[20,8 \%)}\end{array}$ & $\begin{array}{l}\geq 2,00 \mathrm{~cm} \\
\geq 2,50 \mathrm{~cm} \\
\geq 3,00 \mathrm{~cm}\end{array}$ & $\begin{array}{l}0,89 \\
0,93 \\
0,90\end{array}$ \\
\hline $\begin{array}{c}\geq 250 \mathrm{ml} \\
{[15,7 \%)}\end{array}$ & $\begin{array}{l}\geq 2,00 \mathrm{~cm} \\
\geq 2,50 \mathrm{~cm} \\
\geq 3,00 \mathrm{~cm}\end{array}$ & $\begin{array}{l}0,85 \\
0,94 \\
0,74\end{array}$ \\
\hline $\begin{array}{c}\geq 300 \mathrm{ml} \\
{[11,9 \%)}\end{array}$ & $\begin{array}{l}\geq 2,00 \mathrm{~cm} \\
\geq 2,50 \mathrm{~cm} \\
\geq 3,00 \mathrm{~cm}\end{array}$ & $\begin{array}{l}0,81 \\
0,93 \\
0,96\end{array}$ \\
\hline
\end{tabular}

* A diferença nos percentuais refere-se a valores missing.

$\mathrm{Na}$ análise da concordância dos métodos subjetivos para diagnóstico delinfedema, verificou-se concordância simples variando entre $70 \%$ e $75 \%$ (tabela 5). A melhor concordância observada foi entre o relato em prontuário médico e 0 relato de paciente $(75 \%)$, apresentando resultado similar à pior concordância observada entre os métodos objetivos (74\%).

Tabela 5 - Concordância dos métodos subjetivos utilizados no diagnóstico de linfedema

\begin{tabular}{|c|c|c|}
\hline $\begin{array}{l}\text { Mátodos } \\
\text { prenalänxia (\%) }\end{array}$ & Mútodos & $\begin{array}{c}\text { Concordancia } \\
\text { simples }\end{array}$ \\
\hline \multirow{2}{*}{$\begin{array}{l}\text { Relcto de edema broço } \\
\qquad(30,5 \mathrm{sw})\end{array}$} & Relcto em prontuório & 0,75 \\
\hline & Sintomas sugestivos & 0,71 \\
\hline $\begin{array}{c}\text { Sintomas sugestives } \\
\qquad(23,1 \%)\end{array}$ & Reloto em prontudirio & 0,70 \\
\hline
\end{tabular}

* A diferença nos percentuais refere-se a valores missing.

Ao comparar os métodos subjetivos e os melhores métodos objetivos utilizados para o diagnóstico de linfedema, observou-se concordância simples variando entre $71 \%$ e $81 \%$ (tabela 6). A concordância simples mais alta foi observada entre a perimetria $=2,5 \mathrm{~cm}$ e 0 relato em prontuário (81\%). 
Tabela 6 - Concordância entre os métodos objetivos e subjetivos para diagnóstico de linfedema.

\begin{tabular}{|c|c|c|}
\hline $\begin{array}{c}\text { Métodos objetivos } \\
\text { prevalënolo (Ko) }\end{array}$ & $\begin{array}{c}\text { Métodos subjefivos } \\
\text { prevalencia [\%] }\end{array}$ & $\begin{array}{c}\text { Concordáncic } \\
\text { timples }\end{array}$ \\
\hline \multirow{3}{*}{$\begin{array}{l}\text { Voluma estimodo } \\
2200 \mathrm{ml}(20,8 \%)\end{array}$} & $\begin{array}{c}\text { Relato em } \\
\text { prontuário }(16,23)\end{array}$ & 0,79 \\
\hline & $\begin{array}{l}\text { Relato de edemo } \\
\qquad\{30,5 \%\}\end{array}$ & 0,76 \\
\hline & $\begin{array}{c}\text { Sintomas sugastivos } \\
\qquad \mid 23,1 \%\}\end{array}$ & 0,71 \\
\hline \multirow{3}{*}{$\begin{array}{c}\text { Perimetria } \\
\geq 2,50 \mathrm{~cm}[17,8 \%]\end{array}$} & $\begin{array}{c}\text { Relato em } \\
\text { prontuárie }\{16,2 \%\}\end{array}$ & 0,81 \\
\hline & $\begin{array}{l}\text { Relato de edema } \\
\qquad\{30,5 \% \mid\end{array}$ & 0,79 \\
\hline & $\begin{array}{c}\text { Sintomas sugestivos } \\
\qquad 23,1 \%\}\end{array}$ & 0,74 \\
\hline
\end{tabular}

* A diferença nos percentuais refere-se a valores missing.

$\mathrm{Na}$ análise da validade do diagnóstico através da perimetria com ponto de corte $=2,0 \mathrm{~cm}$, foi observada sensibilidade de 0,96 e especificidade de 0,86, com 35\% de falso-positivos e $1 \%$ de falso-negativos. Adotando 0 ponto de corte da perimetria $=2,5 \mathrm{~cm}$, foi observada sensibilidadeinferior $(0,74)$ emelhor especificidade $(0,98)$, com $10 \%$ de falso-positivos e $6 \%$ de falso-negativos. Ao utilizarmos o ponto de corte de $3,0 \mathrm{~cm}$ na perimetria, a sensibilidade foi ainda inferior $(0,56)$, com pouca melhora na especificidade $(0,99)$, apresentando $6 \%$ de falsopositivos e $10 \%$ de falso-negativos.

Entre os métodos subjetivos, o relato em prontuário apresentou sensibilidade de 0,39 e especificidade de 0,90, com $50 \%$ de falso-positivos e $15 \%$ de falso-negativos. 0 relato da paciente de edema no braço mostrou melhor sensibilidade $(0,65)$ e especificidade inferior $(0,78)$, com $56 \%$ de falso-positivos e $11 \%$ de falso-negativos. Os sintomas sugestivos de edema apresentaram pior sensibilidade $(0,35)$ e pouca melhora na especificidade $(0,80)$, aumentando o percentual de falso-positivos para $68 \%$ e de falso-negativos para $18 \%$ (tabela 7 ).
A associação entre a sensibilidade e o percentual de falso- positivos para o diagnóstico de linfedema foi analisada através da curva ROC. 0 melhor ponto de corte foi de $2,0 \mathrm{~cm}$, com área abaixo da curva ROC de 0,91 , sendo classificado como excelente segundo a classificação proposta por Tape 31. 0 s pontos de corte de 2,5 e 3,0 cm foram, respectivamente, classificados como bom e satisfatório $(0,87$ e 0,77).

\section{DISCUSSÃO}

N este estudo, a prevalência de linfedema variou entre 11,9 e $30,7 \%$, segundo os métodos diagnósticos e os pontos de corte utilizados. O utros autores também encontraram diferenças nas prevalências observadas em uma mesma população, denotando a importância da padronização dos instrumentos de diagnóstico, de forma a proporcionar a comparação entre os estudos ${ }^{32-35}$.

Em estudo realizado com 100 pacientes submetidas à linfadenectomia para câncer de mama sem radioterapia axilar, a incidência de linfedema, após 1 ano de seguimento, foi de $10 \%$, considerando 0 aumento de $200 \mathrm{ml}$ entre os membros, determinado através do volumêtro optoeletrônico ${ }^{36}$. Edwards ${ }^{33}$, através da medição do volume de água deslocada em 201 mulheres, com seguimento médio de 37 meses, encontrou prevalência de linfedema de $11 \%$, considerando uma diferença igual ou maior que $10 \%$ entre os membros. 0 mesmo autor, considerando apenas as queixas subjetivas de linfedema, encontrou prevalência de $23,4 \%$. Kwan et $a^{37}$, examinaram 57 pacientes com sintomas no braço ipsilateral ao câncer de mama, e 55 sem sintomas, considerando como linfedema, diferença igual ou maior que $200 \mathrm{ml}$ entre os membros, medida através do volume de água deslocada. Esses autores observaram prevalências de linfedema de 17,5\% e 7\%, respectivamente, para cada grupo. O utro estudo,

Tabela 7 - Validade entre os métodos utilizados para diagnóstico de linfedema, considerando padrão-ouro o volume estimado do membro $\geq 200 \mathrm{ml}$.

\begin{tabular}{lccccc}
\hline \multicolumn{1}{c}{ Métodos objetivos } & Prevalência (\%) & Sensibilidade & Especificidade & VPP & VPN \\
\hline Perimetria $\geq 2,00 \mathrm{~cm}$ & 30,7 & 0,96 & 0,86 & 0,65 & 0,99 \\
Perimetria $\geq 2,50 \mathrm{~cm}$ & 17,8 & 0,74 & 0,98 & 0,90 & 0,94 \\
Perimetria $\geq 3,00 \mathrm{~cm}$ & 12,4 & 0,56 & 0,99 & 0,94 & 0,90 \\
\hline \multicolumn{1}{c}{ Métodos subjetivos } & & & & & \\
\hline Relato em prontuário & 16,2 & 0,39 & 0,90 & 0,50 & 0,85 \\
Relato de edema & 30,5 & 0,65 & 0,78 & 0,44 & 0,89 \\
Sintomas sugestivos 2 ou + & 23,1 & 0,35 & 0,80 & 0,32 & 0,82 \\
\hline
\end{tabular}

* A diferença nos percentuais refere-se a valores missing. 
realizado através de questionário, verificou prevalência de $21,3 \%$ de relato de linfedema entre as mulheres submetidas à linfadenectomia e à radioterapia axilar, em comparação a $12,4 \%$ entre aquelas submetidas apenas à linfadenectomia axilar $(p=0,04)$, com seguimento médio de 3,3 anos $^{38}$. M eric et al ${ }^{39}$ estudaram, retrospectivamente, 294 mulheres submetidas à cirurgia conservadora e à radioterapia, com seguimento médio de 89 meses; o linfedema foi avaliado através da circunferência dos membros, considerando como critério para diagnóstico a diferença de 2,00 cm; os autores observaram uma prevalência de 13,6\%. Em outro estudo com 332 mulheres, onde também foi utilizada a circunferência do membro para diagnóstico do linfedema, considerou-se, como requisito para diagnóstico, uma diferença igual ou maior que 2,00 $\mathrm{cm}$, a prevalência observada de linfedema foi de $14 \%$ em braço e de $11 \%$ em ante-braço ${ }^{40}$. Em um estudo, 128 mulheresquehaviam sido submetidas à cirurgia para câncer de mama há 20 anos foram entrevistadas por telefone e a presença de linfedema foi determinada com base em medida de circunferência efetuada pela entrevistada, sendo observada uma prevalência de $49 \%{ }^{41}$.

$\mathrm{Edwards}^{33}$, utilizando como método de diagnóstico o relato de edema e o volume do membro (considerando linfedema o volume $>10 \%$ ), observou prevalência de linfedema de $11 \%$ e $23 \%$, respectivamente. 0 autor não relatou a confiabilidade e validade dos métodos utilizados e atribuiu a diferença observada ao ponto de corte utilizado e ao tempo transcorrido entre as duas aferições. Em nosso estudo, a prevalência do linfedema através do relato de edema foi de 30,5\% e, através do volume estimado, foi de $20,8 \%$, sendo observada uma concordância simples de 0,76 entre ambos.

É possível que o relato de sintomas sugestivos de linfedema, como sensação de pele esticada e sensação de peso e o relato de "inchaço" no membro, possa ser atribuído a outras alterações provocadas pela cirurgia, como a parestesia no trajeto do nervo intercostobraquial, a intercostobraquialgia, e a trombose linfática superficial, o que dificulta o diagnóstico de linfedema efetuado com base nesse método. Entretanto, observou-se que, nos casos mais evidentes, havia anotação da presença de linfedema, em prontuário, ou seja, quanto maior 0 volume do membro, maior a possibilidade de que 0 linfedema seja percebido pelo profissional, uma vez que as medidas de circunferência não faziam parte da rotina do exame físico no período do estudo.

Entre os métodos objetivos, a concordância simples variou entre $74 \%$ e $96 \%$, considerando diferentes pontos de corte. 0 volume estimado $\geq 300 \mathrm{ml}$ e a perimetria $\geq$
3,00 cm apresentaram melhor concordância, o que pode ser explicado pela facilidade em se fazer o diagnóstico, quanto maior for a discrepância entre os membros. A perimetria utilizando o ponto de corte $\geq 2,5 \mathrm{~cm}$, mostrou ser um método com excelente concordância ao compararmos com o volume estimado de $200 \mathrm{ml}, 250$ $\mathrm{ml}$ ou $300 \mathrm{ml}(0,93 ; 0,94 ; 0,93$; respectivamente). Resultado divergente foi publicado por $M$ egens et $a{ }^{35}$, onde a mensuração do linfedema foi obtida através do volume direto (água deslocada) e do volume indireto (a partir das circunferências) em 25 mulheres sob risco de desenvolver linfedema após tratamento para câncer de mama, concluindo em seu estudo que o volume obtido tanto pela circunferência, como através da água deslocada possuem excelente confiabilidade teste reteste, entretanto com pouca concordância entre métodos.

A melhor concordância entre os métodos subjetivos, foi similar à pior concordância entre os métodos objetivos. Segundo a American Cancer Society ${ }^{42}$, pode haver discordância entre as queixas subjetivas de linfedema e os achados ao exame físico. Entretanto, a discordância não exclui a importância de ambos os métodos, pois as queixas subjetivas da paciente podem preceder a capacidade de detectar alterações objetivas do linfedema e a apresentação única de sintomas deve induzir o médico a efetuar avaliações objetivas, orientações preventivas e seguimentos mais freqüentes, visando o diagnóstico precoce. Recomendação semelhante foi publicada pelo Comitê de Câncer de M ama do Canadás, sendo relatado que os sintomas de peso, rigidez ou inchaço no braço afetado precisam ser considerados pel os médicos nas avaliações de seguimento de câncer de mama, podendo servir como guia para o diagnóstico precoce de linfedema e para o referenciamento ao especialista com vistas à imediata intervenção.

A escolha de um padrão-ouro para diagnóstico de linfedema é fundamental na análise da validade dos instrumentos utilizados para mensuração do linfedema. C asley-Smtih ${ }^{29}$ considerou que tanto a circunferência, como o volume do membro, eram métodos igualmente válidos. Kissin et al ${ }^{32}$ relataram ser a perimetria inacurada, pois varia de acordo com o grau de constrição dos tecidos moles que o observador emprega, sendo preferível medir 0 volume de forma direta. M egens et $a^{35}$ reportaram que tanto a circunferência, como o volume de água deslocada, possuem excelente reprodutibilidade interna.

Entre os métodos subjetivos, o relato de edema apresentou sensibilidade de 0,65 , com inclusão de apenas $44 \%$ das pacientes que apresentavam diagnóstico de linfedema com base no padrão-ouro 
adotado. Kissin et $a^{32}$, verificando a validade de métodos subjetivos (relato de edema), e objetivos (perimetria $>4 \mathrm{~cm}$ e volume $>200 \mathrm{ml}$ ) em pacientes submetidas a tratamento para câncer de mama, observaram queo volume do membro apresentou melhor sensibilidade, incluindo $96 \%$ dos pacientes com linfedema subjetivo e apresentou apenas $9 \%$ de falsopositivos. Esse achado divergente poderia ser atribuído ao ponto de corte utilizado para a perimetria $(4,0)$ e à obtenção do volume do membro pela forma direta.

A mensuração do linfedema através da perimetria foi realizada por uma única pesquisadora, treinada e habilitada para tal função, que desconhecia a presença dos sintomas subjetivos de linfedema. A entrevista antecedeu o exame físico, sendo realizada no mesmo dia, e por outro profissional. D urante a coleta de dados do prontuário, era desconhecido o resultado do exame físico e da entrevista. Assim, a introdução de viés por conhecimento prévio do diagnóstico de linfedema, nesta casuística, seria pouco provável.

Kosier et $a^{34}$, preconizam o desenvolvimento de protocolos de seguimento para as pacientes submetidas a tratamento para câncer de mama, buscando identificar os sintomas precursores do linfedema.

Em nosso meio, se espera uma freqüência el evada de linfedema, devido ao tratamento mais radical do câncer, acarretado pelo diagnóstico tardio da doença. Sendo assim, o método de diagnóstico de linfedema para essa população deve ser de fácil aplicação, baixo custo, rápido e seguro. Além disso, o diagnóstico deve favorecer a introdução precoce de condutas terapêuticas específicas, visando a minimizar as seqüelas e prevenir a cronicidade do linfedema.

Com base nos achados deste estudo, e considerando as características da população brasileira e do sistema de saúde do país, acreditamos que o melhor método a ser incorporado para a definição do linfedema seja a perimetria, com ponto de corte de $2,5 \mathrm{~cm}$. Esse método, além de ser de mais fácil execução, apresentou a melhor relação entre sensibilidade e especificidade. $A$ diferença de 2,0 cm entre os membros constitui um indicativo de linfedema em fase inicial, chamando a atenção para a necessidade de instituir condutas que visam prevenir 0 quadro de cronicidade e a instalação de seqüelas físicas, funcionais, psicológicas e sociais advindas do diagnóstico tardio desta patologia. Todavia, devido ao alto percentual de indivíduos classificados como falso-positivos a partir da utilização desse ponto de corte, se o objetivo for a identificação apenas daqueles casos que necessitam de condutas mais agressivas, deve-se adotar um ponto de corte mais elevado.

\section{CONCLUSÃO}

O s métodos subjetivos não se mostraram adequados para o diagnóstico de linfedema na população estudada. Este fato não exclui sua importância, mas devem ser mais bem investigados quanto à possibilidade destes sintomas serem precedentes ao aparecimento do linfedema, servindo como base para o diagnóstico e intervenção precoce.

O s métodos que apresentaram melhor concordância foram o volume estimado do membro $\geq 300 \mathrm{ml}$ e a perimetria $\geq 3,00 \mathrm{~cm}$. A perimetria de $2,5 \mathrm{~cm}$, apresentou excelente reprodutibilidade com 0 volume estimado do membro independente dos pontos de corte utilizados (200, 250 e $300 \mathrm{ml})$.

Considerando como padrão-ouro o volume estimado do membro $\geq 200 \mathrm{ml}$, a perimetria com ponto de corte de $2,5 \mathrm{~cm}$ foi 0 método com melhor relação sensibilidade/especificidade $(S=0,74 ; E=0,98)$.

\section{REFERÊN CIAS}

1. Instituto $N$ acional de $C$ âncer; $M$ inistério da Saúde. Estimativas de câncer no Brasil. Rio de Janeiro (Brasil): IN CA; 2003.

2. Paz WA, Paim SP, M ello GL, Rangel KK, Christo RC, Komatsuzaki F. Linfadenectomia axilar: análise retrospectiva de 470 casos de câncer de mama em diferentes estádios clínicos. Rev Bras M astol. 2001;11(2):57-65.

3. PalmeiraH T, N aidu S, JuaçabaS, FerreiraM V, Rabenhorst SH . Características anatomopatológicas e dados epidemiológicos de pacientes com câncer de mama submetidas a tratamento cirúrgico na M aternidadeEscola Assis Chateaubriant. Rev Bras M astol. 2002;12(1):31-4.

4. Instituto $N$ acional deCâncer; $M$ inistério da Saúde. Registro hospitalar decâncer. D ados das unidades do IN CA. Rio de Janeiro (Brasil): IN CA; 2000.

5. H arris SR, H ugi M R, O livotto IA, Levime M ; Steering Committee for $C$ lincal Practice $G$ uidelines for the $C$ are and Treatment of Breast $\mathrm{C}$ ancer. Clinical practice guidelines for the care and treatment of breast cancer: 11. Lymphedema. CM AJ. 2001;164:191-9.

6. N ational Breast $\mathrm{C}$ ancer $\mathrm{C}$ entre. Lymphedema: prevalence, risk factors and management: a review of research [cited $1999 \mathrm{M}$ ar]. Availablefrom: http://www.nbcc.org.au

7. Stanton AWB, Svensson WE, M ellor RH, Peters AM, Levick J R, M ortimer PS. D ifferences in lymph drainage between swollen and non-swollen regions in arms with breast-cancer-related lymphoedema. Clin Sciences. 2001;101:131-40.

8. Passik S, M cD onalds M. Psychosocial aspects of upper extremity lymphedema in woman treated for breast 
carcinoma. C ancer. 1998;83 Suppl:2817-20.

9. Velanovich V, Szymanski W. Q ualit of life of breast cancer patients with lymphedema. Am J Surg. 1999;177:184-8.

10. The Lymphedema Association of Australian. H ow can lymphedema betreated? [cited $1999 \mathrm{M}$ ar]. Avaliablefrom: http://www.lymphoedema.org.au

11. Andrade M FC. Linfedema. In: Pitta GBB, Castro AA, Burihan E. Angiologiaecirurgia vascular: guiailustrado. Rio deJaneiro: U N CISAL/ECM AL\& LAVA; 2003 [citado em out 2003]. D isponível em: http://www.lava.med.br/livro.

12. Petrek JA, Lerner R. Linfedema. In: D oenças da mama. H arris], M orrow M, O sborneK. 2a ed. Rio de Janeiro: M edsi; 2002.

13. Petrek J, H eelan M . Incidence of breast carcinoma: related lymphedema. C ancer. 1998;83 Suppl:2776-81.

14. Consensus D ocument of the International Society of Lymphology Executive Committee. The diagnosis and treatment of peripheral lymphedema. Lymphology. 1995;28:113-7.

15. N ational C ancer Institute. Lymphedema[ [cited 2000 Jun]. Avaliablefrom: http://www.cancernet.nci.nih.gov

16. Buncel $H, M$ irolo BR, $H$ ennessy JM, Ward LC, Jones LC. Post-mastectomy lymphoedema treatment and measurement. M ed J Aust. 1994;161(2):125-8.

17. Foldi E. T hetreatment of lymphedema. Cancer. 1998;83 Suppl:2833-4.

18. Camargo M C, M arx AG . Reabilitação física no câncer de mama. São Paulo: Roca; 2000.175p.

19. Rockson SG, M iller LT, SenieR, Brennan M J, C adey-SmithJR, Foldi E, et al. American Cancer Society LymphedemaWorkshop. Workgroup III: diagnosisand management of lymphe dema. C ancer. 1998;83 (12 Suppl American): 2882-5.

20. Leduc A, Leduc O . D renagem linfática: teoriae prática. 2a ed. São Paulo: M anole; 2000. 66p.

21. N ational Lymphedema N etwork. Lymphedema [cited 2000 M ay]. Available from: http://www.lymphnet.org

22. Gerber L. A review of measures of lymphedema. $C$ ancer. 1998;83 Suppl:2803-4.

23. H oe AL, Iven D, Royle GT, Taylor I. Incidence of arm swelling following axillary clearance for breast cancer. $\mathrm{Br}$ J Surg. 1992;79:261-2.

24. Kargearges J R, M ark BE, Stikeleather SJ, Worrell TW. C oncurrent validity of upper-extremity volumeestimates: comparison of calculated volume derived from girth measurements and water displacement volume. Phys Ther. 2003;83(2):134-45.

25. Boland R, Adams R. D evelopment and evaluation of a precision forearm and hand volumeter and measuring cylinder. J H and Ther. 1996;9(4):349-58.

26. Sander AP, H ajer N M , H emenway K, M iller AC. U pperextremity volumemeasurementsin women with lymphedema: a comparison of measurements obtained via water displacement with geometrically determined volume. Phys Ther. 2002;82(12):1201-12.

27. Kaulesar Sukul DM, den H oed PT, Johannes EJ, Van $D$ older $R$, BendaE. D irect and indirect methods for quantification of leg volume: comparison between water displacement volumetry, the disk model method and thefrustum sign model method, using the correlation coefficient and limits of agreement. J Biomed Eng. 1993;15:477-80.

28. M arkowisk J, W ilcon J, H elm P. Lymphedemaincidence after specific postmastectomy therapy. Arch Phys M ed Rehabil. 1981;62(9):449-52.

29. Casley-Smith J. M easuring and representing peripheral oedema and its alterations. Lymphology. 1994;27:56-70.

30. Szklo M, N ieto J. Epidemiology: beyond the basics. An Aspen Publication; 2000.

31. TapeT G . Interpreting diagnostic tests [cited $20020 \mathrm{ct}$ ]. Available from: http://freebooks4doctors.com/fb/ spec16.htm

32. Kissin M W, Q uerci della RovereG , Easton D, Westbwery $G$. Risk of lymphoedema following thetreatment of breast cancer. BrJ Surg. 1986;73:580-4.

33. EdwardsT. Prevalence and etiology of lymphedema after breast cancer treatment in Southern Tasmania. Aust N Z J Surg. 2000;70:412-8.

34. Kosir M A, Rymal C, Koppolu P, H ryniuk L, D arga L, D u $W$, et al. Surgical outcomes after breast cancer surgery: measuring acutelymphedema.J Surg Res. 2001;95(2):147-51.

35. M egens AM, H arris SR, Kim-Sing C, M C Kenzie DC. $M$ easurement of upper extremity in woman after axillary dissection for breast cancer. Arch Phys M ed Rehabil. 2001;82(12):1639-44.

36. D uff M , H ill AD, M CG real G, Wal sh S, M CD ermott EW, $\mathrm{O}^{\prime} \mathrm{H}$ iggins $\mathrm{N}$ J. Prospectiveevaluation of themorbidity of axillary clearance for breast cancer. $\mathrm{Br} J$ Surg. 2001;88(1):114-7.

37. Kwan W, Jackson J, Weir LM , D ingee C, M CG regor G, $O$ livotto IA. Chronic arm morbidity after curative breast cancer treatment: prevalence and impact on quality of life. J Clin O ncol. 2002;20(20):4242-8.

38. Yap KP, M cC ready D R, N arod S, M anchul LA, Trudeau $M$, Fyles $A$. Factorsinfluencing arm and axillary symptoms after treatment for nodenegativebreast carcinoma. $C$ ancer. 2003;97(6):1369-75.

39. M eric F, BuchholzTA, M irza N Q, Vlastos G, AmesFC, Ross M I. Long-term complicationsassociated with breastconservation surgery and radiotherapy. Ann Surg O ncol. 2002;9(6):543-9.

40. Voogd AC, Ververs JM, Vingerhoets AJ, Roumen RM , Coebergh JW, C rommelin M A. Lymphoedemaand reduced shoulder function asindicators of quality of lifeafter axillary lymph nodedissection for invasive breast cancer. Br J Surg. 2003;90(1):76-81.

41. Petrek JA, SenieRT, Peters M , Rosen PP. Lymphedemain a 
cohort of breast carcinoma survivors 20 years after diagnosis. Cancer. 2001;92(6):1368-77.
42. American C ancer Society LymphedemaW ork Group [cited 19990 ct]. Availablefrom: htpp://www.cancer.org 